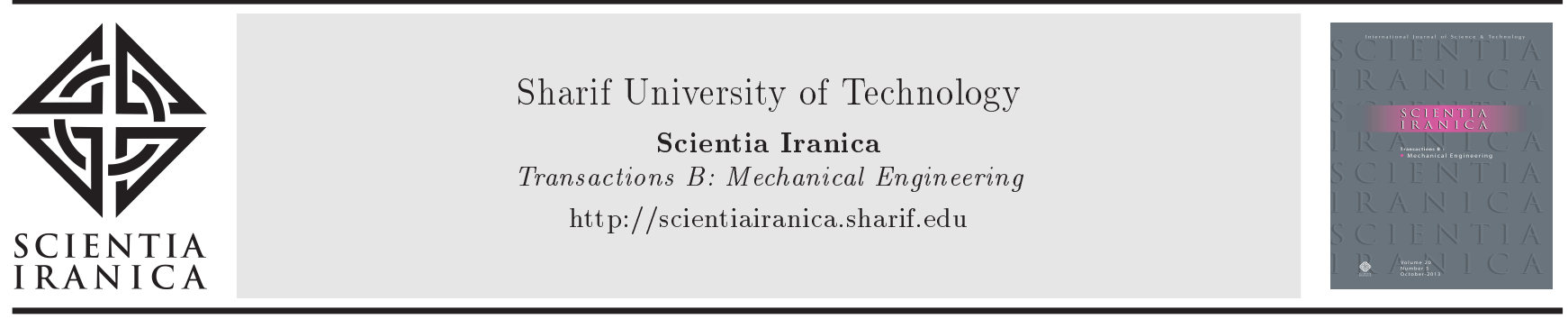

\title{
Proposing a high-efficiency dielectrophoretic system for separation of dead and live cells
}

\author{
H. Shayestehpour, K. Nassiri Nazif, A.M. Soufi, and M.S. Saidi* \\ School of Mechanical Engineering, Sharif University of Technology, Tehran, Iran.
}

Received 14 September 2015; received in revised form 2 April 2016; accepted 8 April 2017

\section{KEYWORDS}

Dielectrophoresis;

Live and dead cells;

Separation;

Micro-electrodes.

\begin{abstract}
Recently, electrode-based Dielectrophoresis (eDEP) has been used for particle manipulation by means of triangular electrodes. In this theoretical and numerical study, a microchannel with quarter-of-ellipse electrodes is presented and a detailed comparison with triangular electrodes is provided. Electric field, resultant DEP force, and particle trajectories for each microchannel are evaluated by means of COMSOL Multiphysics 4.4. Afterwards, focusing and separation efficiencies of the systems are assessed and compared. Finally, separation efficiency of our proposed model for live and dead cells is compared with that of our previous model published in the literature [1]. It is demonstrated that our proposed new model has higher lateral DEP force, responsible for cell separation, than the previous triangular-electrode model had. This feature is reflected in the $96 \%$ focusing efficiency for 10-micron particles and $100 \%$ separation efficiency for live and dead mammalian cells.
\end{abstract}

(C) 2018 Sharif University of Technology. All rights reserved.

\section{Introduction}

The advent of micro/nano technologies has enabled us to develop systems capable of efficiently manipulating, separating, monitoring, and treating the biological cells [2-5]. There are several mechanical, electrical, thermal, optical, acoustic, magnetic, and chemical phenomena within microfluidic systems [6-9]. Among them, dielectrophoresis, which is based on the dielectric properties of particles relative to the suspension fluid, can be efficiently used in order to separate dead and live cells. The above-mentioned properties are closely relevant to structure and composition of the

\footnotetext{
*. Corresponding author. Tel.: +98 2166165558 ; Fax: +982166000021

E-mail addresses: hamedshayeste@gmail.com (H. Shayestehpour); koosha.nassiri@gmail.com (K. Nassiri Nazif); am.soufi@yahoo.com (A.M. Soufi); mssaidi@sharif.edu (M.S. Saidi)
}

particles and are crucial to operate their direction of motion $[9,10]$.

Dielectrophoretic (DEP) force is generated as a result of the electric field gradient. In electrodebased DEP (eDEP), which utilizes different geometry figures and dispositions of photolithographically patterned electrodes, the non-uniform electric field within the microchannel created by electrodes results in particle movement by causing a DEP force. Therefore, disparate voltages of electrodes at different frequencies can manipulate particles throughout the microchannel. Recently, there has been a significant increase in the use of eDEP due to the evolution of high-precision micronsized electrodes using photolithography methods [11].

Dielectrophoresis has been utilized for detection, manipulation, separation, and characterization of cells [12-15], bacteria [16-18], virus [19,20], parasites [21,22], DNA molecules [23,24], and cell organelles $[25,26]$.

For the first time, Pohl and Hawk (1966) investigated DEP separation of live and dead cells in 
a stationary system [27]. The study of stationary DEP cell separation systems was then pursued by a number of investigators $[19,28,29]$. Becker et al. (1995) successfully separated breast cancer cells (MDA 231) from healthy cells by means of castellated electrodes and frequency dependency of DEP force to reach the trapping effect [7].

To circumvent the process challenges faced in stationary systems, continuous flow DEP separation techniques were offered by investigators [30,31]. Continuous particle separation can also be accomplished by both confining and branching particle streams. Confining particles into an infinitesimal volume is a principal technique in many microdevices and has been widely utilized $[32,33]$.

Hemmatifar et al. numerically simulated a continuous flow eDEP microfluidics device for separation of live and dead cells comprising four triangular microelectrodes patterned on the top and bottom of the channel [1]. However, they did not investigate the efficiency of this system with regard to the separation of live and dead mammalian cells.

In this study, we have proposed new highly efficient, eDEP micro-particle focusing as well as cell sorting microsystems, which are based on the study by Hemmatifar et al [1]. The design introduced herein features a new type of electrodes, which generate higher gradients in the electric field and, therefore, higher DEP force for particle manipulation. We have performed numerical experiments and, based on them, we have predicted separation efficiency of our system to be 100 percent along with its extremely high focusing efficiency and, thus, its superiority over our previous DEP micro-particle focusing and cell separating system [1]. Simulation of the separation of the average live and dead mammalian cells in the range of 1-10 microns in diameter is performed at the optimum configuration of the applied voltages. Finally, the efficiency of the separation process as well as the focusing efficiency of live cells is calculated.

\section{Theoretical background}

For simplification, we have considered biological cells as spherical particles. The time-averaged DEP force for a homogenous spherical particle is given as follows [34]:

$$
F_{\mathrm{DEP}}=2 \pi r^{3} \varepsilon_{\text {medium }} \operatorname{Re}\left[f_{\mathrm{CM}}(\omega)\right] \nabla E_{\mathrm{rms}}^{2},
$$

where $r$ and $\varepsilon_{\text {medium }}$ are the radius of the particle and the permittivity of the suspending medium, respectively, and $\operatorname{Re}\left[f_{\mathrm{CM}}(\omega)\right]$ and $E_{\mathrm{rms}}$ are the real part of the Clausius-Mossotti (CM) factor and the root-meansquare value of the electric field, respectively. CM factor, which represents polarizability of the system, can be calculated as follows:

$$
f_{\mathrm{CM}-\text { cell }}=\frac{\varepsilon_{\text {cell }}^{*}-\varepsilon_{\text {medium }}^{*}}{\varepsilon_{\text {cell }}^{*}+2 \varepsilon_{\text {medium }}^{*}},
$$

where $\varepsilon^{*}$ is the complex permittivity defined as:

$$
\varepsilon^{*}=\varepsilon-\frac{i \sigma}{\omega},
$$

where $i=\sqrt{-1}, \sigma$ and $\omega$ are the electric conductivity and the angular frequency of the electric field, respectively.

The single shell [35] model was used to model the mammalian cells. The single shell model is an approach to model protoplasts (spherical particles in which it is possible to identify a cytoplasm and a lossless membrane) (see Figure 1).

$\mathrm{CM}$ function for the live mammalian cells is borrowed from Jones, 2003 [34] as:

$$
\begin{aligned}
& f_{\mathrm{CM}}(\omega)= \\
& -\frac{\omega^{2}\left(\tau_{1} \tau_{m}-\tau_{c} \tau_{m}^{*}\right)+i \omega\left(\tau_{m}^{*}-\tau_{1}-\tau_{m}\right)-1}{\omega^{2}\left(\tau_{c} \tau_{m}^{*}+2 \tau_{1} \tau_{m}\right)-i \omega\left(\tau_{m}^{*}+2 \tau_{1}+\tau_{m}\right)+2},
\end{aligned}
$$

where $c_{m}$ and $R$ are the effective capacitance of the membrane and the radius of the cell, respectively, and $\tau_{m}=c_{m} R / \sigma_{c}$ and $\tau_{c}=\varepsilon_{c} / \sigma_{c}$ are the time constants; subscript $c$ refers to the cytoplasm. It is assumed that the conductance of the membrane $g_{m}$ is negligible (lossless membrane). It should be noted that $\tau_{1}=\varepsilon_{1} / \sigma_{1}$ and $\tau_{m}^{*}=c_{m} R / \sigma_{1}$.

Supposing that a dead cell has an irreversibly permeable membrane, its $\mathrm{CM}$ factor equation becomes [35]:

$$
f_{\mathrm{CM}}(\omega)=\frac{\varepsilon_{c}-\varepsilon_{1}-i\left(\sigma_{2}-\sigma_{1}\right) / \omega}{\varepsilon_{c}+2 \varepsilon_{1}-i\left(\sigma_{2}+2 \sigma_{1}\right) / \omega} .
$$

Deionized (DI) water, whose properties are $\varepsilon_{1}=78 \varepsilon_{o}$, is chosen as a very resistive medium (subscript 0 refers to vacuum). We have followed the practice of Khoshmanesh et al. [5] in using DI water. The important properties of the medium for the simulations reported herein are density, viscosity, and electric

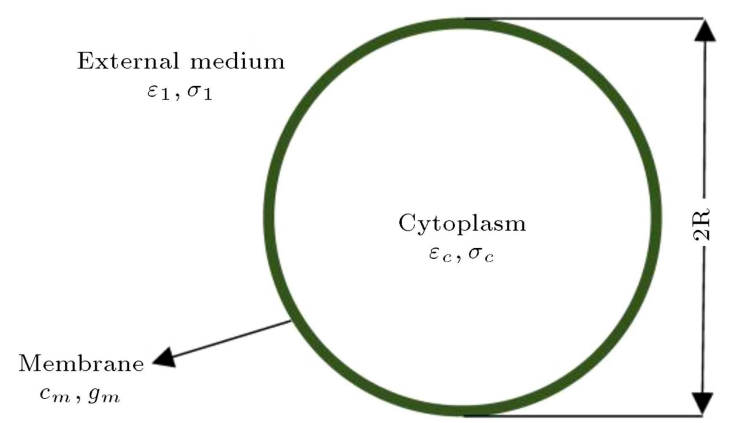

Figure 1. Protoplast model parameters. 


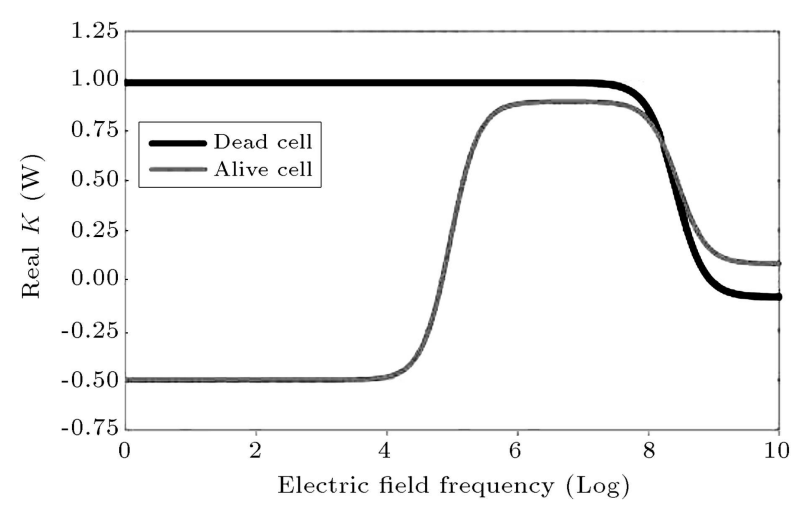

Figure 2. The CM function for dead and live mammalian cells.

permittivity, which do not vary very much if some small amounts of ions are introduced in the solution to make the system isotonic. Please notice that the cells are modelled as particles and, therefore, cell viability is not an issue here. Thus, we believe that using DI water for simulations is a compromise between addressing all the details of isotonic medium and losing small amount of accuracy. follows:

The average values of the cell parameters are as

- Cytoplasm:

$$
\varepsilon_{c}=60 \varepsilon_{0}, \quad \sigma_{c}=0.5 \mathrm{~S} / \mathrm{m}, \quad R=2.0 \mu \mathrm{m} .
$$

- Loss-less membrane:

$$
C_{m}=1.0 \mu \mathrm{F} / \mathrm{cm}^{2}, \quad g_{m}=0 .
$$

CM factors for dead and live cells as a function of electric field frequency are calculated using the aforementioned values and method. Real part of CM factors for frequencies less than $10 \mathrm{e} 4 \mathrm{~Hz}$ for dead and live cells is 1 and -0.5 , respectively (see Figure 2) [35].

\section{Design and simulation}

\subsection{Layout of the microchannel}

Lateral sorting method is used for the separation of dead and live cells. Due to their positive and negative CM factors, dead and live cells move toward the sidewalls and centerline of the channel, respectively. This method can be utilized here to semi-continuously manipulate the particles.

Figure 3 shows the isometric and top views of the channel used by Hemmatifar et al. [1]. Also, the microchannel proposed herein is shown in the figure, with four quarter-of-ellipse electrodes. The dimensions of the microchannels are $1000 \times 260 \times 100(-500<x<$ $500,20<y<120,-130<z<130 \mu \mathrm{m})$ micrometers and the electrodes are placed on the top (A and $\mathrm{B}$ ) and bottom ( $\mathrm{C}$ and $\mathrm{D})$ surfaces of the microchannels

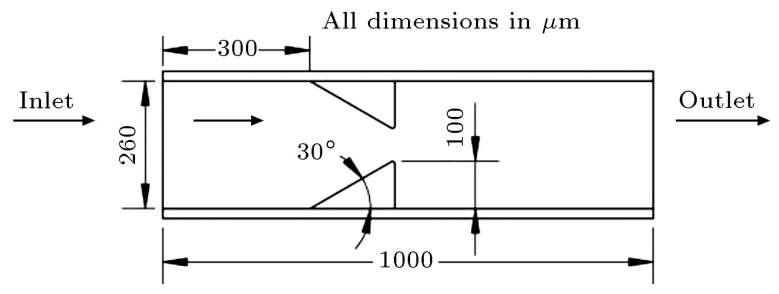

(a)

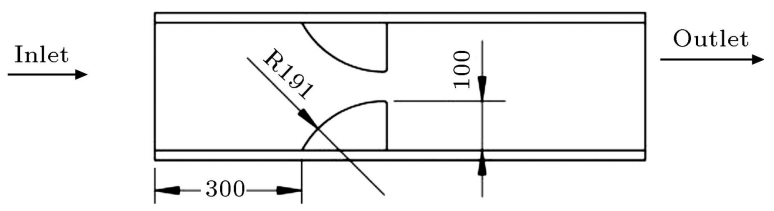

(b)

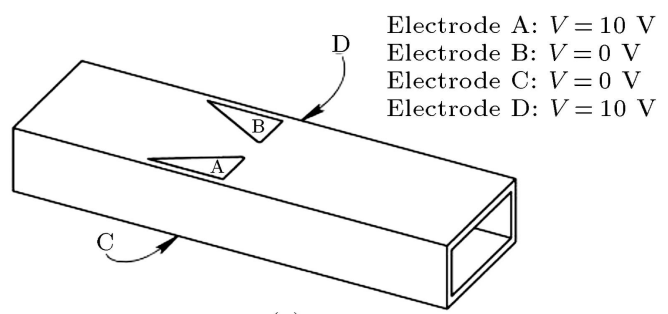

(c)

Figure 3. (a) Two-dimensional layout of Hemmatifar et al.'s triangular electrodes. (b) Layout of the proposed quarter-of-ellipse electrodes in the channel. (c) Three-dimensional view of the microchannel with four microelectrodes. Electrodes A and B are at the top, whereas $\mathrm{C}$ and $\mathrm{D}$ are located at the bottom of the microchannel. All dimensions are in millimeters.

for complete three-dimensional particle manipulation. Two microelectrodes (A and $\mathrm{D}$ ) have voltage, $V$, while the other two (B and $\mathrm{C}$ ) are grounded.

\subsection{Method of simulation}

COMSOL Multiphysics 4.4 CFD code was utilized in order to simulate and assess the focusing, cell sorting, and separation efficiency of the proposed microchannel. Three modules were included in this simulation: laminar flow, electric current, and particle tracking in fluid flow.

For modelling the flow, the prescribed inlet velocity of water was set at 100 micrometers/second. Since the reservoirs were of very low depth and in contact with the atmospheric air, the pressure at the outlet was also assumed to be atmospheric. With water density, $\rho=998.2 \mathrm{~kg} / \mathrm{m}^{3}$, and dynamic viscosity, $\mu=0.001 \mathrm{~kg} / \mathrm{m} / \mathrm{s}$, the Reynolds number was equal to 0.010 . Therefore, the flow was creeping and the hydrodynamics entry length was so short that the flow would certainly become fully developed before reaching the electrodes. The flow no-slip boundary condition was set on microchannel walls.

For the electric current module, the initial voltage value for the whole microchannel was set to zero. As described above, electrodes $\mathrm{A}$ and $\mathrm{D}$ had a voltage 
of magnitude, $\mathrm{V}$, whereas the electrodes $\mathrm{B}$ and $\mathrm{C}$ were grounded. For the other surfaces of the channel including inlet, outlet, bottom, top, and sidewalls, the electrical current was set to zero as the boundary condition (see Figure 3).

The channel geometry was discretized into unstructured, tetrahedral cells. Grid clustering was performed near the edges of the electrodes, where the gradient of the electric field was supposed to be high. To study grid independency, four meshes (fine, finer, extra fine, and extremely fine) were constructed. Figure 4 shows the magnitude of the electric field along the line $(z=0, y=50,-500<x<500 \mu \mathrm{m})$ in finer grid of COMSOL Multiphysics 4.4. Investigation of the magnitude of the electric field demonstrated that the error associated with the use of finer meshes instead of extremely fine ones was less than 1.5 percent. Therefore, finer mesh was used in order to decrease the computational costs.

Table 1 shows the parameters that were set in the numerical simulations.

To model the particle motion, only drag and dielectrophoretic forces were taken into account. As the flow was creeping, the drag force on spherical cells was approximated by the Stokes' law given as follows:

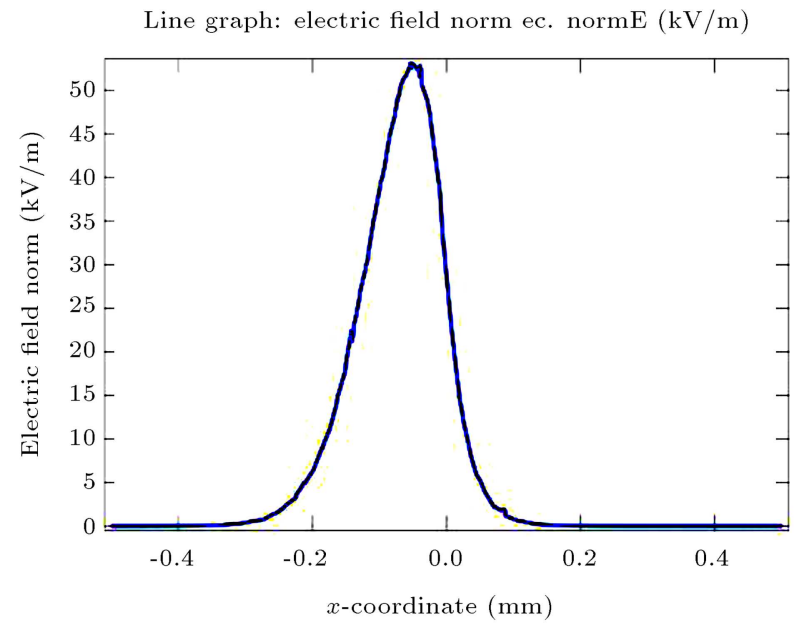

Figure 4. Magnitude of the electric field of our model along the line $(z=0, y=50,-500<x<500 \mu \mathrm{m})$ in finer grid of COMSOL Multiphysics 4.4.

$$
F_{\text {drag }}=6 \pi \mu r V_{\text {rel }} \text {, }
$$

where $V_{\text {rel }}$ is the velocity of the particle relative to fluid flow and $r$ is the outer radius of the particle. Assuming that the concentration of particles in the fluid flow was not high, the interaction of particles with each other was not considered in this simulation.

Considering the particle tracking in fluid flow physics, a particle diameter range of 1-10 microns (average diameter of most biological particles) was considered in our simulations. 65 live cells and 65 dead cells were separately tracked in two simulations released from uniform positions at the inlet $(5 \times 13)$. Live cells concentration and trapping the dead cells resulted in their separation in the microchannel under investigation.

\section{Results and discussion}

Figure 5 shows the results with regards to the part of steady state in simulation and exhibits flow velocity plot and distribution of electric field magnitude overlaying vector plot of $\frac{\partial E^{2}}{\partial y} \hat{e}_{y}+\frac{\partial E^{2}}{\partial z} \hat{e}_{z}$ in three $y-z$ sections along the $x$ axis when $10 \mathrm{~V}$ is applied on two electrodes in crossed charging configuration. Partial derivative of $E^{2}$ with respect to $y$ and $z$ variables is responsible for the $y$ - and $z$-components of DEP force; therefore, it plays a key role in the lateral (along the $z$ axis) and vertical (along the $y$ axis) movements of the particles in the channel. The $\frac{\partial E^{2}}{\partial y}$ in all sections is negative in the zone of $0<y<130$ microns, and is positive in the zone of $130<y<260$ microns. Consequently, considering the negative value of $\mathrm{CM}$ factor of live cells, they are expected to travel to the $y=130$-micron line. The $\frac{\partial E^{2}}{\partial z}$ in all sections is centripetal around the $y=130$-micron line. In fact, a secondary concentration occurs in $\mathrm{Z}$ direction for all configurations. This is due to the negative value of $\frac{\partial E^{2}}{\partial z}$ in the zone of $0<z<50$ microns and its positive value in the zone of $50<z<100$ microns. These forces make a concentrated particle stream at the outlet. The dead cells with positive value of CM factor, however, are expected to be stuck near the electrodes where $\frac{\partial E^{2}}{\partial y} \hat{e}_{y}+\frac{\partial E^{2}}{\partial z} \hat{e}_{z}$ is maximum. These predictions are in agreement with the particle tracking simulation

Table 1. Parameters considered in simulation.

\begin{tabular}{cccc}
\hline Parameter of simulation & Value & Domain selection & Material \\
\hline Medium density & $998.2 \mathrm{kgm}^{-3}$ & Electrodes & Silver \\
Medium dynamic viscosity & $0.001 \mathrm{kgm}^{-1} \mathrm{~s}^{-1}$ & Microchannel body & Acrylic Plastic \\
Inlet velocity & $0.0001 \mathrm{~m} / \mathrm{s}$ & Medium & Water \\
Outlet pressure condition & $0 \mathrm{~Pa}$ & & \\
Initial value of electric potential of microchannel & $0 \mathrm{~V}$ & \\
Cells density & $1000 \mathrm{kgm}^{-3}$ & \\
\hline
\end{tabular}


Slice: Velocity mangnitude $(\mathrm{m} / \mathrm{s})$; Arrow line: velocity field; Arrow volume: velocity field

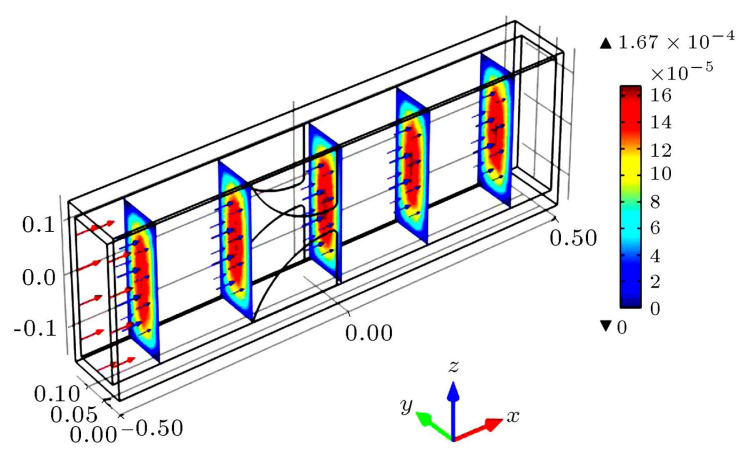

(a)

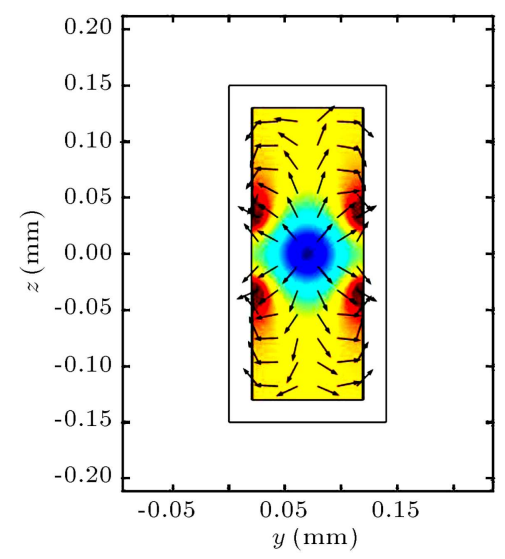

(c)

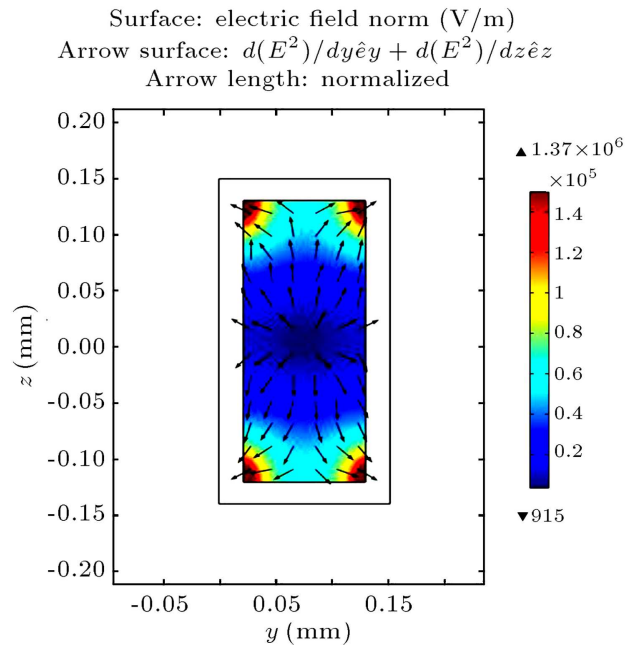

(b)

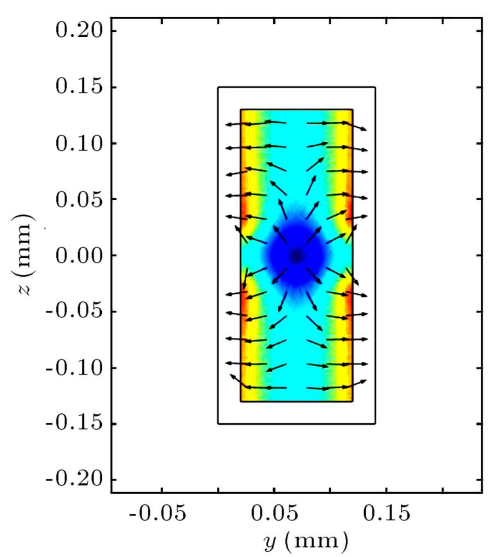

(d)

Figure 5. Steady state results of simulation: (a) Laminar flow field and flow velocity contour in channel, (b) distribution of electric field magnitude overlaying vector plot of $\frac{\partial E^{2}}{\partial y} \hat{e}_{y}+\frac{\partial E^{2}}{\partial z} \hat{e}_{z}$ at the $x=-200 \mu \mathrm{m}$ plane when $10 \mathrm{~V}$ is applied on two electrodes in crossed charging configuration, (c) distribution of electric field magnitude overlaying vector plot of $\frac{\partial E^{2}}{\partial y} \hat{e}_{y}+\frac{\partial E^{2}}{\partial z} \hat{e}_{z}$ at the $x=-100 \mu \mathrm{m}$ plane, and (d) distribution of electric field magnitude overlaying vector plot of $\frac{\partial E^{2}}{\partial y} \hat{e}_{y}+\frac{\partial E^{2}}{\partial z} \hat{e}_{z}$ at the $x=0 \mu \mathrm{m}$ plane.

results at voltages of $2,4,6,8$, and $10 \mathrm{~V}$. It is also demonstrated that the live cells are more concentrated in our proposed designs than in the one suggested by Hemmatifar et al. [1].

Live and dead cells released in uniform position from inlet were tracked. Figures 6 and 7 illustrate the pathline and focusing of 4-micron diameter dead and live cells released from our model's inlet with applied electrode voltage of $10 \mathrm{~V}$ for four consecutive times, respectively. It was noticed that by considering 4micron particles, the dead cells were stuck to the wall around the electrode zone while the live cells were moved forward and concentrated exactly on centerline. For particles with diameters greater than 4 microns, the magnitude of dielectrophoretic force was greater and the focusing and separation efficiencies were maximum. Consequently, the entire results that have been represented specifically are for 4-micron particles.
The focusing efficiency is calculated for different applied voltages as a function of cell size for two electrode patterns. The focusing efficiency is defined as the ratio of particle-free region width at the outlet to the width of the channel. This results in the possibility of comparing it with the focusing efficiency of Hemmatifar et al.'s model. The focusing efficiency graphs are plotted in Figure 8 for applied voltages of $2,4,6,8$, and $10 \mathrm{~V}$. Figure 8 demonstrates that when the applied voltage is $10 \mathrm{~V}$, the focusing efficiency of our newly suggested design and that of Hemmatifar et al.'s [1] model for particle diameters greater than 4 and 6 microns are maximum. In our model, when applied voltage is $8 \mathrm{~V}$, focusing efficiency is close to focusing efficiency of Hemmatifar et al. for $10 \mathrm{~V}$. Thus, the focusing efficiency of our model is significantly greater than that of the Hemmatifar et al.'s model [1].

Figure 9 illustrates the trajectories of 65 released 

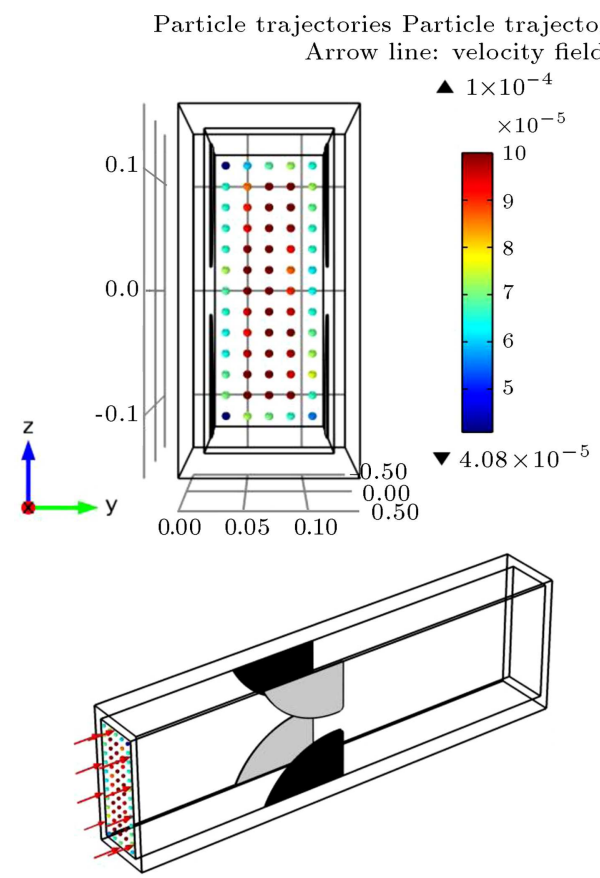

(a)

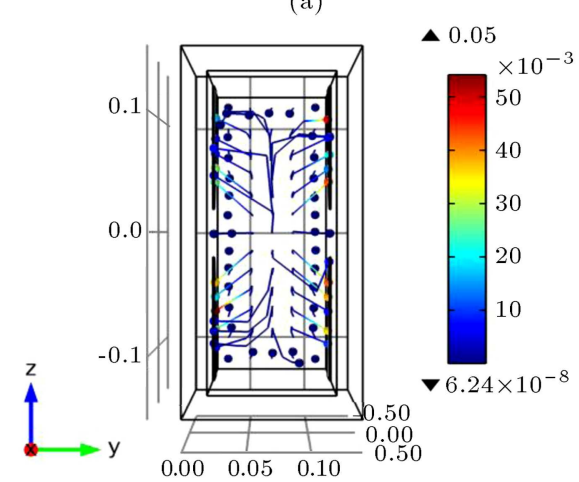

(b)
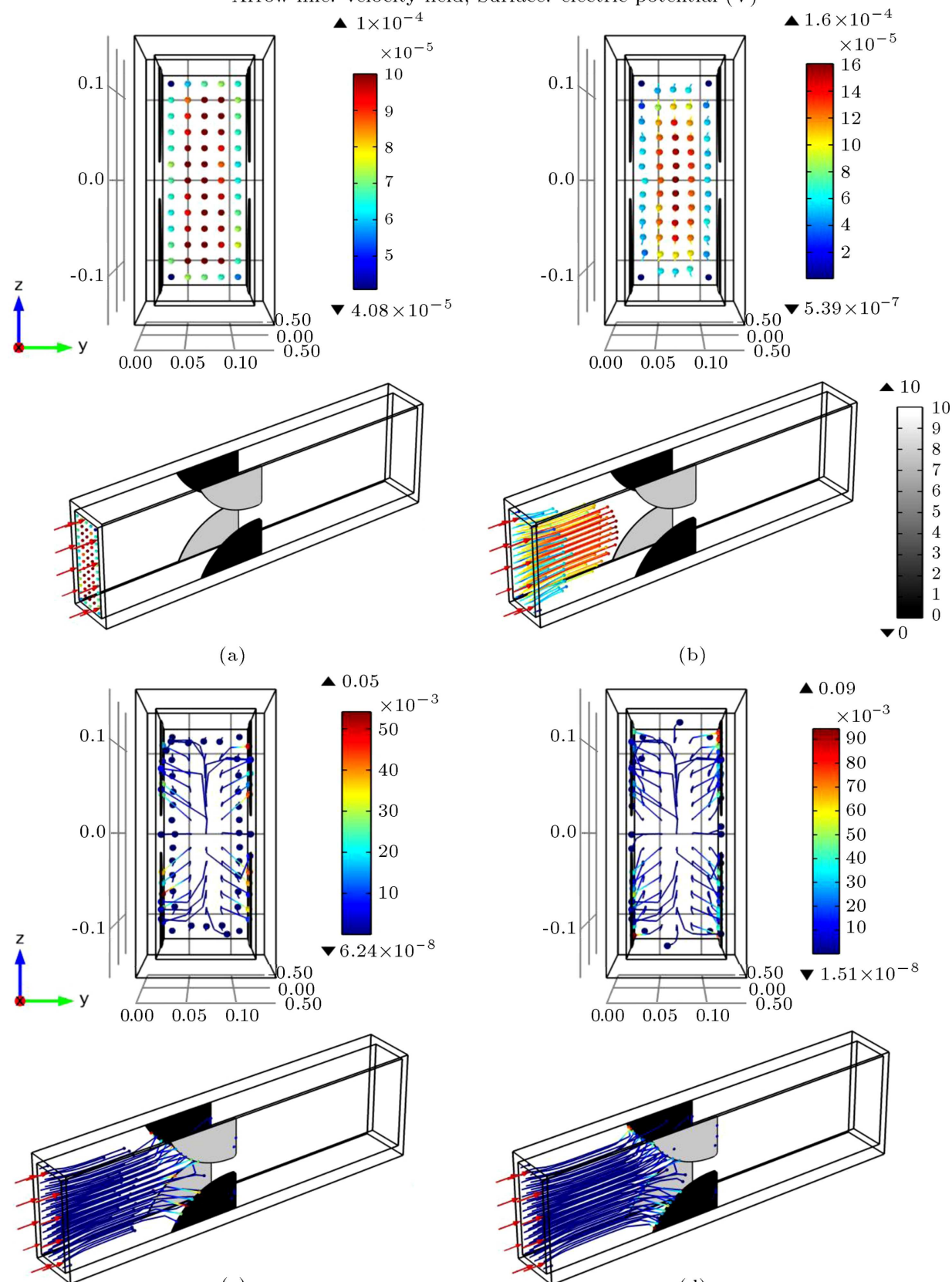

(c)

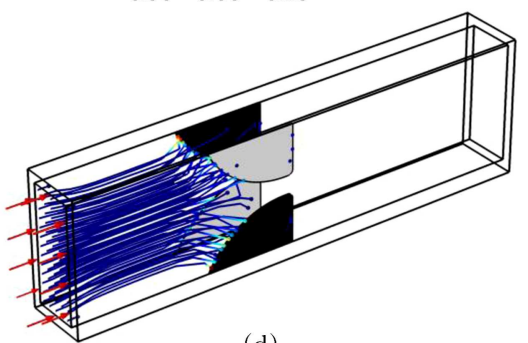

(d)

Figure 6. Distribution and focusing of our model for 65 dead cells with 4-micron diameter released from inlet with applied electrode voltage of $10 \mathrm{~V}$ for four consecutive times. The color of particle trajectories represents particle velocity magnitude $(\mathrm{m} / \mathrm{s}):(\mathrm{a})$ Time $=0 \mathrm{~s}$, (b) time $=2 \mathrm{~s}$, (c) time $=4 \mathrm{~s}$, and (d) time $=7 \mathrm{~s}$.

4-micron diameter cells from uniform positions in the inlet with applied electrode voltage of $10 \mathrm{~V}$. As shown in Figure 9, in the proposed quarter-of-ellipse model, live cells are concentrated on the centerline and all of the dead cells are trapped around the electrodes. In Hemmatifar et al.'s model, live cells are not concentrated on the central line completely; also, some of the dead cells escape from the electrodes.

Simulation results show that separation efficiency of our proposed model is 100 percent for applied voltage of $10 \mathrm{~V}$ and cell diameters greater than 4 microns. This parameter is defined as the ratio of the number of trapped dead cells to the number of released dead cells at the inlet. Therefore, in this range of cell diameters, our proposed model traps all dead cells near the electrodes and concentrates live cells on the center of the channel as they move toward the outlet.

It is worth mentioning that the medium used for simulations was DI water. Real mediums used for cell manipulation usually have some ions deliberately 


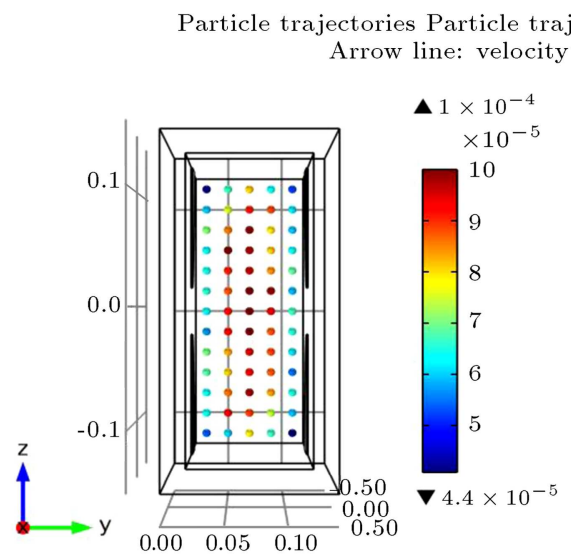

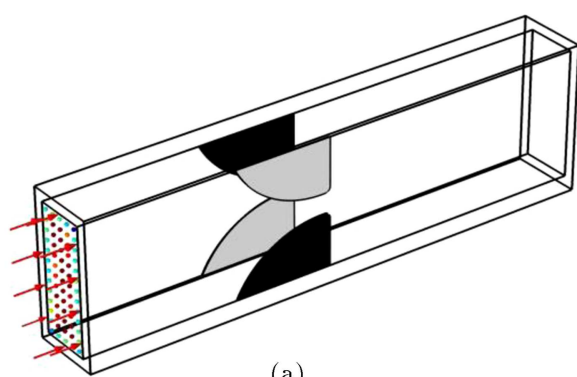

(a)
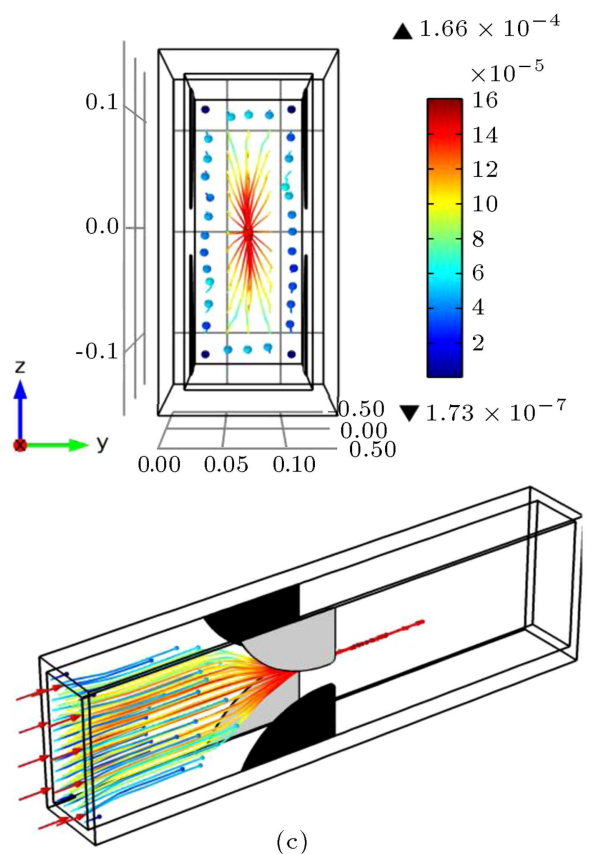
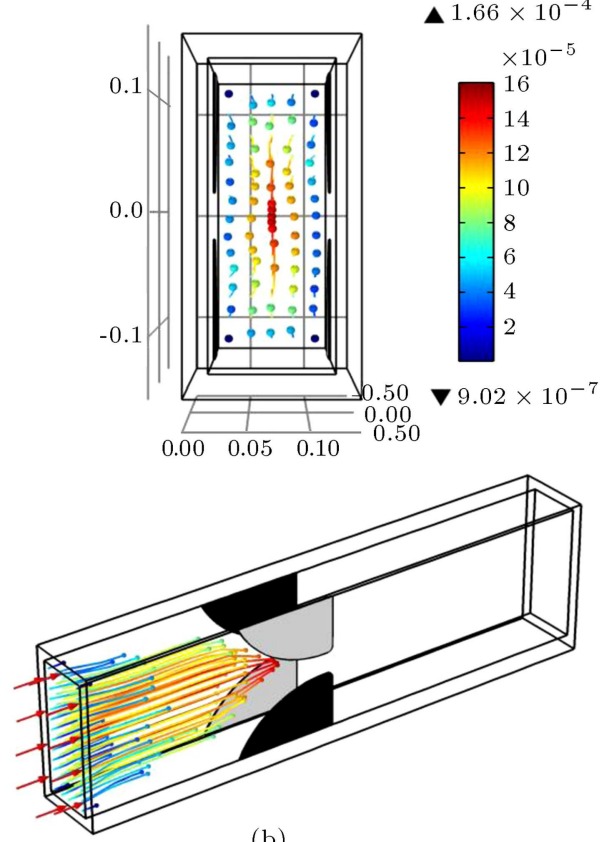

(b)

A $1.66 \times 10^{-4}$
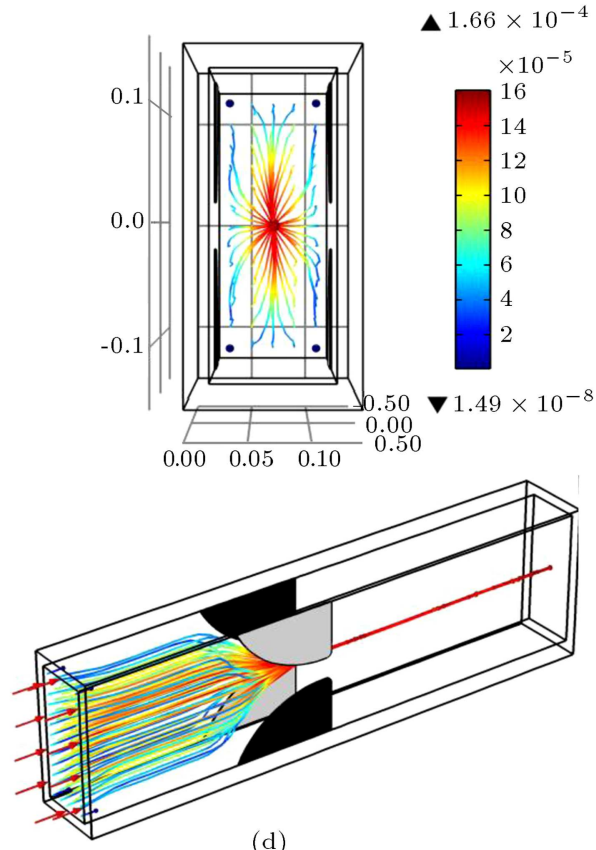

(d)

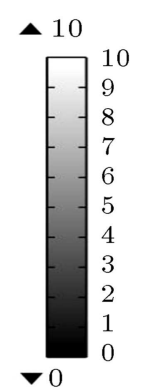




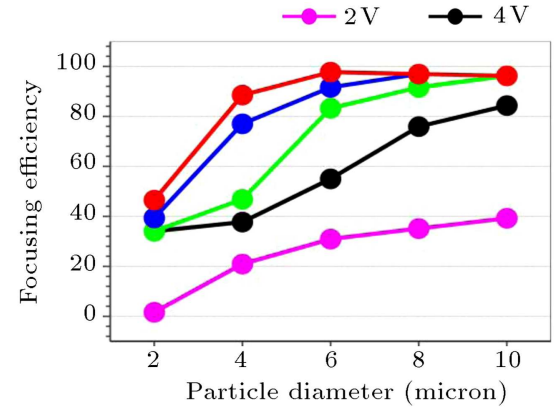

(a)

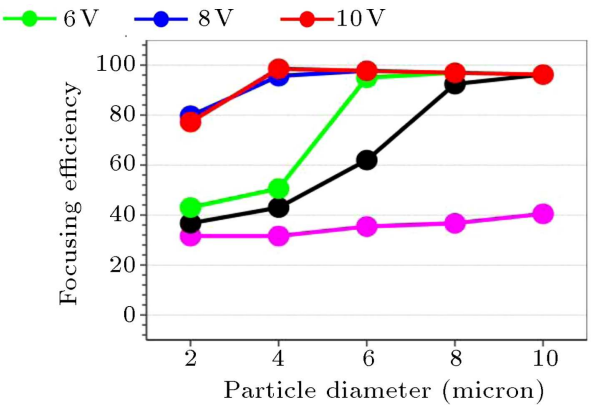

(b)

Figure 8. The focusing efficiency of (a) Hemmatifar et al.'s model and (b) the proposed quarter-of-ellipse model, for different live cell diameters and electrode voltages.

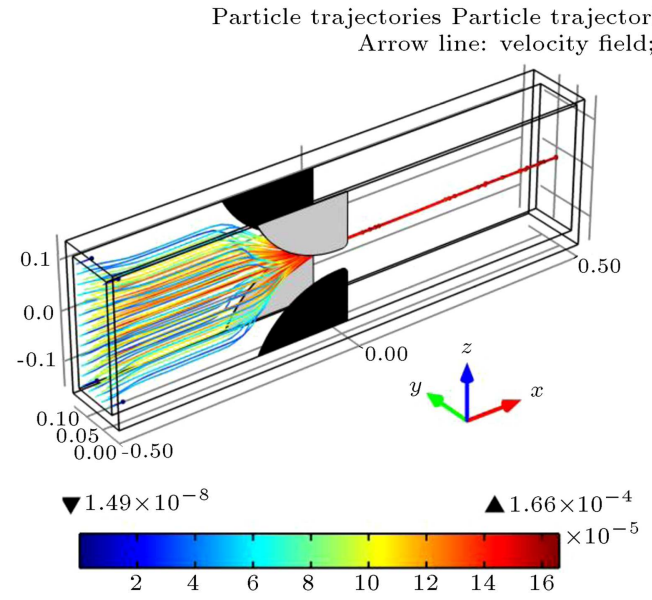

(a)

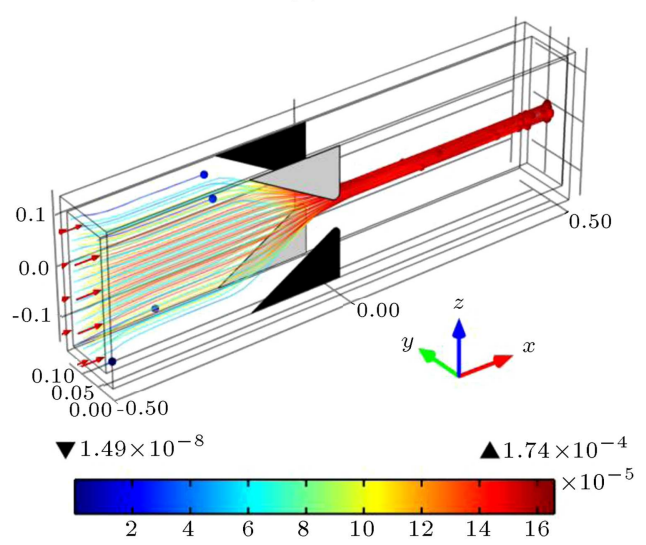

(c)

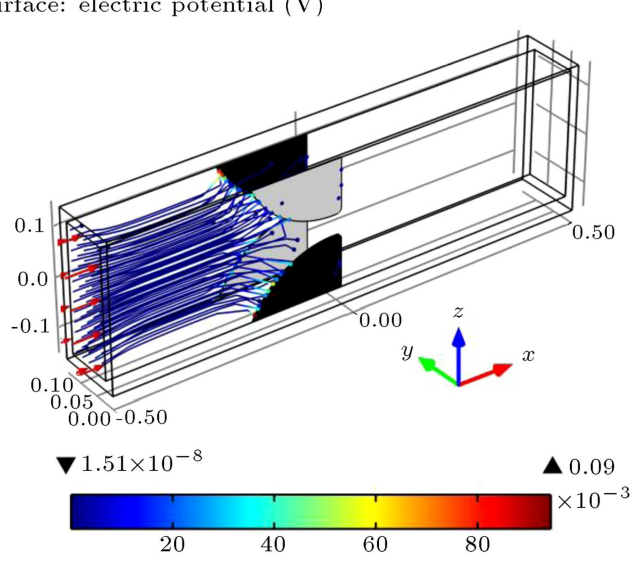

(b)

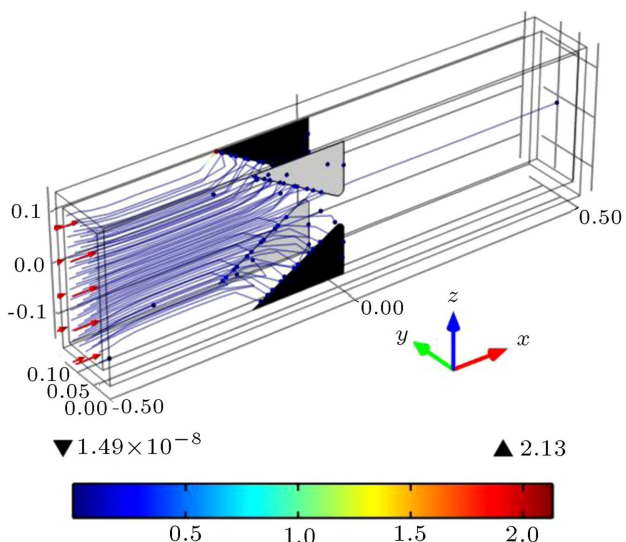

(d)

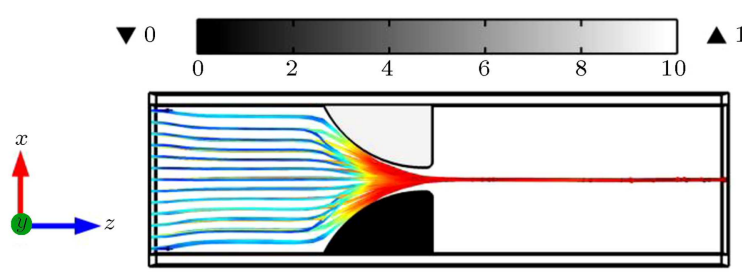

(e)

Figure 9. Trajectories of 65 released 4-micron diameter cells from uniform positions in the inlet with applied electrode voltage of $10 \mathrm{~V}$ beside the path line. The color of particle trajectories represents particle velocity magnitude (m/s): (a) Live cells trajectories for proposed quarter-of-ellipse model, (b) dead cells trajectories for proposed quarter-of-ellipse model, (c) live cells trajectories for Hemmatifar et al., (d) dead cells for Hemmatifar et al., and (e) xz plane of live cells in quarter-of-ellipse model. 
proposed microchannel design has the capability of focusing live mammalian cells as well as separating them from dead ones. We have reported strong electric field gradient generated by the new electrode design compared to Hemmatifar et al.'s [1] design. Similarly, our proposed design can focus on live mammalian cells and separate them from dead mammalian cells with higher efficiency of almost 100 percent.

\section{Acknowledgement}

The authors would like to acknowledge the financial support of Iran National Science Foundation of this work by contract number 90007516 .

\section{References}

1. Hemmatifar, A., Saidi, MS., Sadeghi, A. and Sani, M. "Continuous size-based focusing and bifurcating microparticle streams using a negative dielectrophoretic system", Microfluidics and Nanofluidics, 14(1-2), pp. 265-276 (2013).

2. Baratchi, S., Kanwar, R.K., Khoshmanesh, K., Vasu, P., Ashok, C., Hittu, M., Parratt, A., Krishnakumar, S., Sun, X. and Kanwar, J.R. "Promises of nanotechnology for drug delivery to brain in neurodegenerative diseases", Current Nanoscience, 5(1), pp. 15-25 (2009).

3. Castillo, J., Dimaki, M. and Svendsen, W.E. "Manipulation of biological samples using micro and nano techniques", Integrative Biology, 1(1), pp. 30-42 (2009).

4. El-Ali, J., Sorger, P.K. and Jensen, K.F. "Cells on chips", Nature, 442(7101), pp. 403-411 (2006).

5. Khoshmanesh, K., Kouzani, A.Z., Nahavandi, S., Baratchi, S. and Kanwar, J.R. "At a glance: cellular biology for engineers", Computational Biology Chemistry, 32(5), pp. 315-331 (2008).

6. Andersson, H. and Van den Berg, A. "Microfluidic devices for cellomics: a review", Sensors and Actuators B: Chemical, 92(3), pp. 315-325 (2003).

7. Becker, F.F., Wang, X.B., Huang, Y., Pethig, R., Vykoukal, J. and Gascoyne, P. "Separation of human breast cancer cells from blood by differential dielectric affinity", Proceeding of the National Academy of Sciences, 92(3), pp. 860-864 (1995).

8. Roda, B., Zattoni, A., Reschiglian, P., Moon, M.H., Mirasoli, M., Michelini, E. and Roda, A. "Field-flow fractionation in bioanalysis: a review of recent trends", Analytica Chimica Acta, 635(2), pp. 132-143 (2009).

9. Zhang, C., Khoshmanesh, K., Mitchell, A. and Kalantar-zadeh, K. "Dielectrophoresis for the manipulation of micro/nano particles in microfluidic systems", Analytical and Bioanalytical Chemistry, 396(1), pp. 401-420 (2010).

10. Gascoyne, P.R.C. and Vykoukal, J.V. "Dielectrophoresis-based sample handling in general-purpose programmable diagnostic instruments", Proceedings of the IEEE, 92(1), pp. 22-42 (2004).
11. Morgan, H. and Green, N., AC Electrokinetics: Colloids and Nanoparticles, Research Studies Press (2003).

12. Gray, D.S., Tan, J.L., Voldman, J. and Chen, C.S. "Dielectrophoretic registration of living cells to a microelectrode array", Biosensors and Bioelectronics, 19(7), pp. 771-780 (2004).

13. Hashimoto, M., Kaji, H. and Nishizawa, M. "Selective capture of a specific cell type from mixed leucocytes in an electrode-integrated microfluidic device", Biosensors and Bioelectronics, 24(9), pp. 2892-2897 (2009).

14. Pethig, R. and Talary, M.S. "Dielectrophoretic detection of membrane morphology changes in Jurkat Tcells undergoing etoposide induced apoptosis", IET Nanobiotechnology, 1(1), pp. 2-9 (2007).

15. Urdaneta, M. and Smela, E. "Multiple frequency dielectrophoresis", Electrophoresis, 28(18), pp. 31453155 (2007).

16. Hoettges, K.F., Dale, J.W. and Hughes, M.P. "Rapid determination of antibiotic resistance in E, coli using dielectrophoresis", Physics in Medicine Biology, 52(19), pp. 6001-6009 (2007).

17. Moon, H.S., Nam, Y.W., Park, J.C. and Jung, H.I. "Dielectrophoretic separation of airborne microbes and dust particles using a microfluidic channel for realtime bioaerosol monitoring", Environmental Science and Technology, 43(15), pp. 5857-5863 (2009).

18. Sanchis, A., Brown, A.P., Sancho, M., Martinez, G., Sebastian, J.L., Munoz, S. and Miranda, J.M. "Dielectric characterization of bacterial cells using dielectrophoresis", Bioelectromagnetics, 28(5), pp. 393401 (2007).

19. Morgan, H., Hughes, M.P. and Green, N.G. "Separation of submicron bioparticles by dielectrophoresis", Biophysical journal, 77(1), pp. 516-525 (1999).

20. Morgan, H., Sun, T., Holmes, D., Gawad, S. and Green, NG. "Single cell dielectric spectroscopy", Journal of Physics D: Applied Physics, 40(1), pp. 61-70 (2007).

21. Dalton, C., Goater, A.D., Burt, J.P.H. and Smith, H.V. "Analysis of parasites by electrorotation", Journal of Applied Microbiology, 96(1), pp. 24-32 (2004).

22. Gascoyne, P., Satayavivad, J. and Ruchirawat, M. "Microfluidic approaches to malaria detection", Acta Tropica, 89(3), pp. 357-369 (2004).

23. Hoeb, M., Radler, J.O., Klein, S., Stutzmann, M. and Brandt, M.S. "Light induced dielectrophoretic manipulation of DNA", Biophysical Journal, 93(3), pp. 1032-1038 (2007).

24. Kuzyk, A., Yurke, B., Toppari, J.J., Linko, V. and Torma, P. "Dielectrophoretic trapping of DNA origami", Small, 4(4), pp. 447-450 (2008).

25. Radu, M., Ionescu, M., Irimescu, N., Iliescu, K., Pologea-Moraru, R. and Kovacs, E. "Orientation behavior of retinal photoreceptors in alternating electric fields", Biophysical Journal, 89(5), pp. 3548-3554 (2005). 
26. Uppalapati, M., Huang, Y.M., Jackson, T.N. and Hancock, W.O. "Microtubule alignment and manipulation using AC electrokinetics", Small, 4(9), pp. 1371-1381 (2008).

27. Pohl, H.A. and Hawk, I. "Separation of living and dead cells by dielectrophoresis", Science, 152(3722), pp. 647-649 (1966).

28. Pethig, R., Huang, Y., Wang, X.B. and Burt, J.P.H. "Positive and Negative dielectrophoretic collection of colloidal particles using interdigitated castellated microelectrodes", Journal of Physics D: Applied Physics, 25(5), p. 881 (1992).

29. X.-B. H. Y. H. R. B. J. Wang, "Theoretical and experimental investigations of the interdependence of the dielectric, dielectrophoretic and electrorotational behaviour of colloidal particles", Journal of Physics D: Applied Physics, 26(2), pp. 312-322 (1993).

30. Sano, M.B., Caldwell, J.L. and Davalos, R.V. "Modeling and development of a low frequency contactless dielectrophoresis (cDEP) platform to sort cancer cells from dilute whole blood samples", Biosensors and Bioelectronics, 30(1), pp. 13-20 (2011).

31. Shafiee, H., Sano, M.B., Henslee, E.A., Caldwell, J.L. and Davalos, R.V. "Selective isolation of live/dead cells using contactless dielectrophoresis (cDEP)", Lab on a Chip, 10(4), pp. 438-445 (2010).

32. Fan, S.-K., Huang, P.-W., Wang, T.-T. and Peng, Y.-H. "Cross-scale electric manipulations of cells and droplets by frequency-modulated dielectrophoresis and electrowetting", Lab on a Chip, 8(8), pp. 1325-1331 (2008).

33. Yantzi, J.D., Yeow, J.T.W. and Abdallah, S.S. "Multiphase electrodes for microbead control applications: integration of DEP and electrokinetics for bio-particle positioning", Biosensors and Bioelectronics, 22(11), pp. 2539-2545 (2007).
34. Jones, T., Electromechanics of Particles, Cambridge: Cambridge University Press (2003).

35. Díaz, R. and Payen, S., Biological Cell Separation Using Dielectrophoresis in a Microfluidic Device, Bio and Thermal Engineering Laboratory (2008).

\section{Biographies}

Hamed Shayestehpour received the BS and MS degrees in Mechanical Engineering from Sharif University of Technology, Tehran, Iran in 2015 and 2017, respectively.

Koosha Nassiri Nazif received the BS degree in Mechanical Engineering from Sharif University of Technology, Tehran, Iran, in 2014, and the MS degree in Mechanical Engineering from Stanford University, Stanford, CA, USA, in 2016, where he is currently pursuing the PhD degree in Mechanical Engineering, with a minor in Electrical Engineering.

Amir Mehdi Soufi Enayati received the BS degree in Mechanical Engineering from Sharif University of Technology, Tehran, Iran, in 2016. Since then, he has been studying MS in the same university. His main area of interests is robotics, biomedical engineering, rehabilitation, and biofluidics.

Mohammad Said Saidi is Professor of Mechanical Engineering at Sharif University of Technology, Tehran, Iran. His research interests include modeling and numerical analysis of transport and deposition of aerosol particles, modeling and numerical analysis of biofluids, modeling and numerical analysis of thermalhydraulics in porous media, and microchannels. 